\title{
Model Predictive Control for Building Automation
}

\author{
Peter Bolt ${ }^{1}$, Christian Jaeger ${ }^{1}$, Volker Ziebart ${ }^{1}$, Remo Ritzmann ${ }^{2}$, \\ Olaf Meier ${ }^{2}$, Rudolf Marcel Füchslin ${ }^{1}$ \\ 1 ZHAW School of Engineering, Zurich University of Applied Science, 8401 Winterthur, Switzerland \\ 2 Rino Electronics AG, 8416 Flaach, Switzerland
}

\begin{abstract}
We propose a building HVAC system, integrating local energy production and storage, together with a model based controller. The heating system integrates several local heat production and storage devices and multiple fluid circuits at different temperatures to minimize entropy production through mixing. The controller uses a model of the system and predictive knowledge of demand and weather information to minimize electrical energy import, while maintaining thermal comfort by solving mixed integer optimization problems online. Time-varying and unknown system parameters are estimated and adapted online, using an unscented Kalman filter. The adaptation greatly reduces modeling effort and maintenance cost. The proposed setup is tested in a co-simulation, using a physical (modelica-) model of the building and energy system as well as realistic weather and demand data. Our system delivers nearly seven times more energy in the form of heat, than it needs to import (electrical) energy from external sources.
\end{abstract}

Keywords: PVT, MPC, Unscented Kalman Filter, Modelica.

\section{Introduction}

A HVAC system consists of the physical heating system and - equally important - an optimal control policy. The heating system proposed here integrates several local heat production and storage devices, operating on different time scales, including an ice storage. We use three separate fluid circuits at different temperatures to minimize the entropy arising from mixing temperature levels, and heat pumps to lift heat from one temperature level to another. For the control task, we use the framework of model predictive control (MPC). MPC offers a systematic framework to minimize the electrical import of energy while meeting heat demand. Its usage in building automation has been studied by e.g. (Fux, 2013), (Wimmer, 2004), (Bianchi, 2006), mostly focusing on the task of efficient generation of heat as well as (Sturzenegger, 2014), focusing on the consumer side i.e. reducing heat consumption, while maintaining comfort.

The rising share of electric power that comes from renewable and distributed energy sources fluctuates in a less predictable way, than energy from a "classical" power plant. Multiple demand response programs have been developed and implemented by grid operators during the past years (Ashouri et al., 2015). MPC is well suited to shift the load from a local energy management system towards low-tariff periods, due to the capability to include electrical cost and weather forecasts.

MPC requires a thermodynamic model of the heating system. The amount of work for tuning such a model can be prohibitive (Serale et al., 2018) to the application of MPC in practice. We integrate a framework for on-line estimation of states (Tab. 2) and unknown or time varying parameters (Tab. 4) of the energy supply model, based on an Unscented Kalman Filter (UKF), (Julier et al., 1995), leading to the Parameter-Adaptive Energy Supply Model. Adaptive MPC schemes for building control in which both the unknown parameters and unmeasured states are estimated using UKF or Extended Kalman Filters (EKF), (Peter S. Maybeck, 1979) and (Peter S. Maybeck, 1982), were investigated in (Fux, 2013), (Maasoumy et al., 2014) and (Radecki and Hencey, 2015). The two latter compared EKF and UKF and both concluded, that UKF is superior. A particularly beneficial feature of UKF is the fact that it can handle a nonlinear model, while the model must be linearized for EKF. Detailed investigations of UKF can be found in (Haykin, 2001).

The optimization problem itself is a mixed integer linear optimization problem (MILP), which must be solved once 
per time-step (cf. sec. 3.1). In our case we must handle a MILP with 36 integer and 112 continuous decision variables.

\section{The HVAC system}

The investigated system, integrates a small-scale, grid-connected photovoltaic-thermal collector (PV/T), two heat pumps (HP) charging two thermal storages and supplying energy for surface heating (low temperature), radiator heating (high temperature) and Domestic Hot Water (DHW). The consumers are two single family houses with a heating demand of $\sim 45 \mathrm{kWh} /\left(\mathrm{m}^{2}\right.$ a) and $\sim 100 \mathrm{kWh} /\left(\mathrm{m}^{2}\right.$ a) respectively, denoted SFH45 and SFH100. Fig. 1 shows a simplified schematic of the installation which integrates

- $\quad$ storage devices: ice storage (very long time constant, high capacity, low power, low temperature level) earth tubes, domestic hot water storage, room heating storage (short time constant, low capacity, high power, high temperature)

- local energy production: photovoltaic thermal hybrid (PV/T) collector

- $\quad$ fluid circuits operating at different temperatures levels with heat pumps to lift heat between temperature levels

Unlike geothermal probes, which could also be integrated into the system, the ice storage system does not require planning permission. It provides a reliable, economic and environmentally compatible heat supply to buildings of almost any size, from single-family homes to large residential or commercial buildings. The ice storage is also used for natural cooling in the summer months.

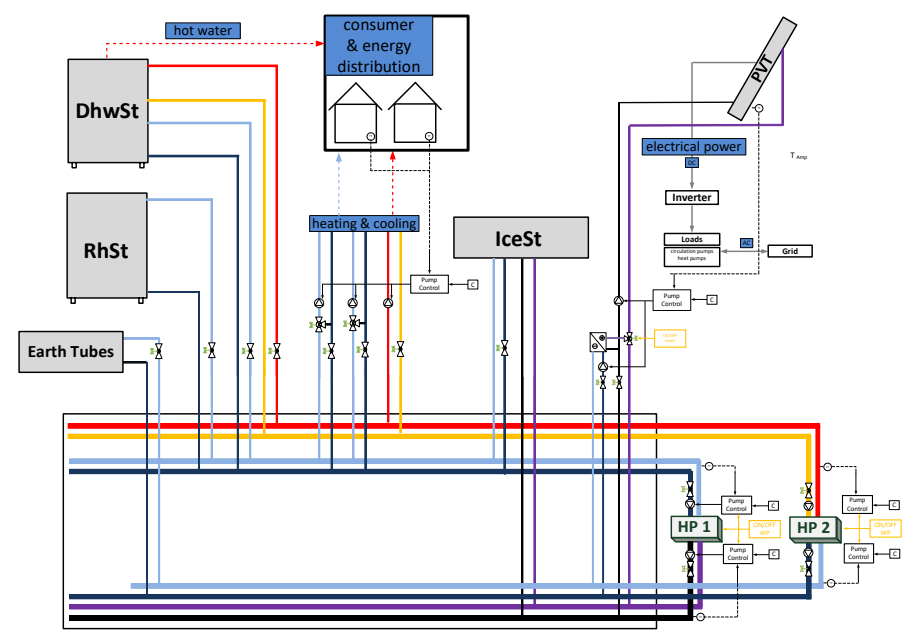

Fig. 1: Simplified schematic of the thermohydraulic system, consisting of fluid circuits, two heat-pumps (HP1, HP2), hot water storages (DhwSt, RhSt), Earth-Tubes, Ice Storage, PV/T collector and two single family homes as consumers.

The consumers, SFH45 and SFH100 along with general boundary conditions such as climate, domestic hot water load and properties of ground and boreholes for the simulation of ground heat exchangers are defined in (Ralf Dott, 2014) and (Haller et al., 2013). We use these definitions for the physical modeling of the energy plant and the building in Modelica. This ensures, that the results are directly comparable to other studies. The boundary conditions, as shown in Tab. 1, will - for the sake of comparability - not be changed, even if a change would further increase the efficiency of the MPC.

PV cell's electrical efficiency exhibits linear temperature dependency (Dubey et al., 2013), (Carigiet et al., 2014). (eq. 1) shows the electrical efficiency of a PV cell. $\eta_{\text {Tref }}$ is the module's electrical efficiency at the reference temperature $T_{\text {ref }}$ and at solar radiation of $1000 \frac{\mathrm{W}}{\mathrm{m}^{2}} \cdot \beta_{\text {ref }}=0.0043 \frac{1}{\mathrm{~K}}$ is the temperature coefficient and $T_{c}$ the actual temperature of the cell.

$$
\eta_{c}=\eta_{\text {Tref }}\left(1-\beta_{\text {ref }}\left(T_{c}-T_{\text {ref }}\right)\right)
$$

In the proposed integration the PV/T panel's main purpose is to regenerate the heat sources (ice storage and earth tubes) during the summer season. Because the thermal energy is led to the cold sources of the heat pumps, the temperature of the PV cells is kept below the reference temperature $T_{r e f}$, which increases electrical efficiency by about $3 \%$.

The main components and some chosen key figures of the heating plant are: 
- Brine to water HP with nominal heat output $3.93 \mathrm{~kW}$ at B0/W35 with a Coefficient of Performance (COP) of 4.65 .

- Brine to water HP with nominal heat output $7.07 \mathrm{~kW}$ at B0/W35 with a COP of 4.65.

- Room heating storage tank (RhSt) 3000 liter, domestic hot water tank (DhwSt) 2200 liter

- Ice storage (IceSt) $84 \mathrm{~m}^{3}$

- Two earth tubes length $60 \mathrm{~m}$

- Photovoltaic thermal hybrid solar collector (PV/T) $40 \cdot 1.641 \mathrm{~m}^{2}$, south facing

\section{Parameter Estimation and Control}

Fig. 2 shows the co-simulation setup. For the simulation environment, shown in the upper half, the open-source and free Modelica (Fritzson and Engelson, 1998) libraries and the Functional Mockup Interface (FMI) were used. Both are results from the IEA EBC project 60 New Generation Computational Tools for Building \& Community Energy Systems (Wetter and van Treeck, 2017). The parameter identification and online optimization scheme are implemented in MATLAB. The optimization problem (including the model) is formulated in MATLAB, using the YALMIP toolbox (Lofberg, 2004) and solved by the Gurobi solver (Gurobi Optimization, LLC, 2018).

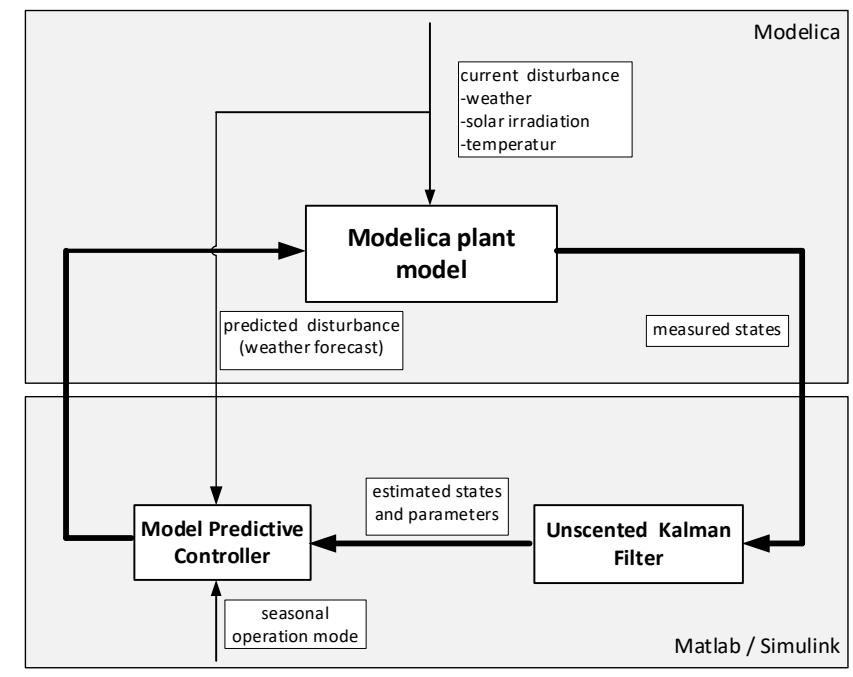

Fig. 2: Co-simulation setup. The upper half represents the simulated environment including heating system and consumers, the lower part represents the controller and state/parameter identification algorithms. The MPC contains a simplified plant model.

Tab. 1: Boundary conditions for simulation according to (Ralf Dott, 2014) and (Haller et al., 2013) for moderate climate of Strasbourg.

\begin{tabular}{llll} 
Parameter & Unit & SFH45 & SFH100 \\
\hline Space heating demand & $\mathrm{kWh} / \mathrm{a}$ & $6{ }^{\prime} 500$ & $14^{\prime} 000$ \\
DHW demand & $\mathrm{kWh} / \mathrm{a}$ & $2{ }^{\prime} 100$ & $2{ }^{\prime} 100$ \\
Heating demand at design conditions & $\mathrm{kW}$ & 4.1 & 7.4 \\
Design supply and return temperature of the heating system & ${ }^{\circ} \mathrm{C}$ & $35 / 30$ & $55 / 45$ \\
\hline
\end{tabular}

\subsection{Model Predictive Control}

(eq. 2) is the Mixed Integer Linear (MILP) Program formulation of the optimization problem

$$
\begin{array}{lll}
J_{0 \rightarrow N}^{*}\left(x_{0}, U_{0 \rightarrow N}\right)= & \max _{U_{0 \rightarrow N}} \sum_{k=0}^{N} q_{w}\left(x_{k}, u_{k}, b_{k}, d_{k}\right) & \\
\text { subject to } & x_{k+1}=F_{w}\left(x_{k}, u_{k}, b_{k}, d_{k}\right), & k=1, \ldots, N-1 \\
& h\left(x_{k}, u_{k}, b_{k}, d_{k}\right) \leq 0, & k=1, \ldots, N-1 \\
& x_{0}=x(0)
\end{array}
$$


with states $x_{k}$, control inputs $u_{k}$, input sequence $U_{0 \rightarrow N}=\left[u_{0}, \ldots, u_{N}\right]$, discrete variables $b_{k}$, disturbances $d_{k}$, such as weather and electricity costs, and the planning horizon $N$. The stage cost function $q_{w}$ (cost for one time step) and the possibly nonlinear system dynamics $F_{w}$, both depending on a set $w$ of system parameters. These parameters are identified with the UKF (see 3.2). The function $h$ encodes the system constraints.

The stage cost function $q_{w}$ (eq. 3) consists of two parts, the energy $E_{e l_{i}}=P_{e l_{i}} d t$ with the energy cost $c_{e}$ and the tracking term with the temperature set-points $T_{s e t_{i}}$ and weights $C_{i}$. The control objective is to keep the temperatures at the top of the storages above $35^{\circ} \mathrm{C}$ and $55^{\circ} \mathrm{C}$ respectively with as little imported electrical energy as possible. Electrical power used in the system, e.g. for operating heat pumps is counted positive, electric power which is produced by the PV/T has a negative sign. Only the positive part of $E_{e l_{i}}$ is considered in (eq. 3), i.e. exporting electricity is cost neutral.

Tab. 2: The MPC (high-level controller) has the following variables. States $x$, control inputs $u$, and disturbance.

\begin{tabular}{|c|c|c|}
\hline Variable & Description & Range/Unit \\
\hline$x_{1}, x_{2}, x_{3}, x_{4}$ & Temperature of the RhSt & ${ }^{\circ} \mathrm{C}$ \\
\hline$x_{5}, x_{6}, x_{7}, x_{8}$ & Temperature of the DwSt & ${ }^{\circ} \mathrm{C}$ \\
\hline$x_{9}$ & Temperature of the PV/T & ${ }^{\circ} \mathrm{C}$ \\
\hline$u_{H P I}$ & HP 1 On/Off & ${ }^{\circ} \mathrm{C}$ \\
\hline$u_{\text {HPITem }}$ & HP 1 supply temperature & $\{0,1\}$ \\
\hline$u_{H P I b}$ & Decision, loading RhSt or DwSt & ${ }^{\circ} \mathrm{C}$ \\
\hline$u_{H P 1}$ & HP 2 On/Off & $\{0,1\}$ \\
\hline$u_{\text {HP2Tem }}$ & HP 2 supply temperature & ${ }^{\circ} \mathrm{C}$ \\
\hline$d_{\text {dist }}$ & Internal gains (unkown) & $\mathrm{W} / \mathrm{m}^{2}$ \\
\hline$d_{T A m b}$ & Ambient temperature & \\
\hline$d_{\text {solar }}$ & Solar radiation & $\mathrm{W} / \mathrm{m}^{2}$ \\
\hline$\left.u_{k}, b_{k}, d_{k}\right)=c_{e} \cdot \max$ & $\left(\sum_{i} E_{e l_{i}}\left(x_{k}, u_{k}, b_{k}, d_{k}\right), 0\right)+$ & (eq. 3) \\
\hline
\end{tabular}

\subsection{State and parameter estimation with an Unscented Kalman Filter}

The accuracy of the model is fundamental for the performance of the MPC. Since each building and heating system is unique, a new model parameter set needs to be identified to represent its behavior. When retrofitting existing buildings, in most cases no Building Energy Model (BEM) will be available, and simulation based parameter tuning is therefore not possible.

Adaptive MPC schemes for building control in which both the unknown parameters and unmeasured states are estimated during operation, using EKF and UKF were proposed in (Fux, 2013), (Maasoumy et al., 2014) and (Radecki and Hencey, 2015). Simulations using these schemes the estimates of the unknown building parameters typically converge after a couple of weeks of operation. The initialization of a Kalman filter's parameters is still delicate and has an impact on the rate of convergence. In order to obtain the best initial parameter value, Maasoumy (Maasoumy et al., 2014) first performed an offline parameter identification based on historical data.

The convergence of EKF/UKF depends on careful choice of design matrices, in particular the initial covariance $P_{0}$, process noise covariance $R_{k}^{v}$ and measurement noise covariance $R_{k}^{n}$. These matrices are often chosen arbitrarily (Schneider and Georgakis, 2013). We propose to use a time-dependent covariance $R_{k}^{v}$ (eq. 5). This reduces the number of parameters to be initialized, since we may set $R_{0}^{v}=\left(\theta^{-1}-1\right) P_{0}$, where $R_{0}^{v}$ and $P_{0}$ are matrices and $\theta$ is a number (Haykin, 2001). For a consistent choice for the initial state covariance $P_{0}$ and initial state $\hat{x}_{0}$, we follow the procedure proposed in (Schneider and Georgakis, 2013).

The UKF was introduced in 1995 (Julier et al., 1995). A refinement for dual state/parameter estimation and for joint state/parameter estimation is given in (Wan and Merwe, 2000). We use the joint UKF framework to simultaneously identify states and parameters, where the signal-state $x_{k}$ and parameter $w_{k}$ vectors are concatenated into a single, joint state vector $\hat{x}_{k}=\left(x_{k} w_{k}\right)^{T}$ which is propagated according to (eq. 4) with control inputs $u_{k}$, process noise $v_{k}$, 
measurement noise $n_{k}$, lumped parameter model $F\left(x_{k}, u_{k}, w_{k}\right)$ and the measurement function $H\left(x_{k}\right)$, which is trivial in our case, since we can directly measure the temperatures.

$$
\begin{aligned}
& \hat{x}_{k+1}=\left(\begin{array}{l}
x_{k+1} \\
w_{k+1}
\end{array}\right)=\left(\begin{array}{c}
F\left(x_{k}, u_{k}, w_{k}\right) \\
w_{k}
\end{array}\right)+v_{k} \\
& y_{k}=H\left(x_{k}\right)+n_{k}=\left(x_{k}\right)+n_{k}
\end{aligned}
$$

Mean and standard deviation of the process noise $v_{k}$ and measurement noise $n_{k}$ can be time varying. The measurement noise covariance $R_{k}^{n}=E\left[n_{k} n_{k}^{T}\right]$ can be set to a constant diagonal matrix (e.g. $R_{k}^{n}=I$ ). The process noise covariance $R_{k}^{v}=E\left[v_{k} v_{k}^{T}\right]$ needs more attention, as it has a key influence in the tracking performance of the algorithm (Haykin, 2001). We set

$$
\begin{aligned}
& R_{k}^{v}=\left(\theta^{-1}-1\right) P_{k} \\
& P_{k}=E\left[\left\{\left(\begin{array}{c}
x_{k} \\
w_{k}
\end{array}\right)-\left(\begin{array}{c}
\hat{x}_{k} \\
\widehat{w}_{k}
\end{array}\right)\right\}\left\{\left(\begin{array}{c}
x_{k} \\
w_{k}
\end{array}\right)-\left(\begin{array}{c}
\hat{x}_{k} \\
\widehat{w}_{k}
\end{array}\right)\right\}^{T}\right]
\end{aligned}
$$

with $\left(\begin{array}{l}\hat{x}_{k} \\ \widehat{w}_{k}\end{array}\right)=E\left[\left(\begin{array}{l}x_{k} \\ w_{k}\end{array}\right)\right]$ and a forgetting factor $\theta \in[0,1]$. This provides an exponentially decaying weighting on past data (Haykin, 2001).

The function $F\left(x_{k}, u_{k}, w_{k}\right)$ can be nonlinear. The UKF propagates the probability distribution of $\hat{x}_{k}$ through $F\left(x_{k}, u_{k}, w_{k}\right)$. To do so, a set $\chi$ of $2 L+1$ sample points with $=\operatorname{dim}(\hat{x})$, called sigma points are chosen, propagated via $\chi_{k+1}=F\left(\chi_{k}, u_{k}\right)$ and the mean and covariance of $\hat{x}_{k+1}$ is calculated. (eq. 6) describes the calculation of sigma points. $\left(\sqrt{(L+\lambda) P_{\hat{x}}}\right)_{i}$ is the $i$ th column of the matrix square root.

\begin{tabular}{l}
\hline Sigma points calculation \\
$\begin{array}{l}\mathcal{X}_{0}=\hat{x} \\
\mathcal{X}_{i}=\hat{x}+\left(\sqrt{(L+\lambda) P_{\hat{x}}}\right)_{i} \quad i=1, \ldots, L \\
\mathcal{X}_{i}=\hat{x}-\left(\sqrt{(L+\lambda) P_{\hat{x}}}\right)_{i-L} \quad i=L+1, \ldots, 2 L\end{array}$
\end{tabular}

The setup for the Unscented Transformation (UT) (eq. 7) is shown below

$$
\begin{aligned}
& \text { UT Setup } \text { (eq. 7) } \\
& \lambda=\alpha^{2}(L+\kappa)-L \\
& W_{0}^{(m)}=\frac{\lambda}{L+\lambda} \\
& W_{0}^{(c)}=\frac{\lambda}{L+\lambda}+1-\alpha^{2}+\beta \\
& W_{i}^{(m)}=W_{i}^{(c)}=\frac{\lambda}{2(L+\lambda)}, \quad i=1, \ldots, 2 L \\
& \gamma=\sqrt{L+\lambda}
\end{aligned}
$$

$\lambda$ is the scaling parameter, which depends on $\alpha, \kappa$ and $L$. The constant $\alpha$ determines the spread of sigma points about the mean $\hat{x}_{k}$. The constant $\kappa$ is a secondary scaling parameter, which we set to $3-L$. $\beta$ is used to incorporate prior knowledge of the distribution. $\beta=2$ is optimal for Gaussian distributions (Haykin, 2001).

Below the UKF Algorithm (eq. 8) is shown, which includes the following main steps:

- $\quad$ Prediction of the model outputs $\hat{y}_{k}^{-}$, based on the model with the current parameters.

- $\quad$ Obtain the measurements $y_{k}$ from sensors

- Update the prediction of the states, based on the errors (measurements-model prediction, $y_{k}-\hat{y}_{k}^{-}$) 


UKF Algorithm

Initialize with:

$$
\begin{aligned}
& \hat{x}_{0}=E\left[x_{0}\right] \\
& P_{0}=E\left[\left(x_{0}-\hat{x}_{0}\right)\left(x_{0}-\hat{x}_{0}\right)^{T}\right]
\end{aligned}
$$

calculate the sigma points:

$$
\mathcal{X}_{k-1}=\left[\begin{array}{lll}
\hat{x}_{k-1} & \hat{x}_{k-1}+\gamma \sqrt{P_{k-1}} & \hat{x}_{k-1}-\gamma \sqrt{P_{k-1}}
\end{array}\right]
$$

Prediction:

$$
\begin{aligned}
& \mathcal{X}_{i, k \mid k-1}^{*}=F\left(\mathcal{X}_{k-1}, u_{k-1}\right) \\
& \hat{x}_{k}^{-}=\sum_{i=0}^{2 L} W_{i}^{(m)} \mathcal{X}_{i, k \mid k-1}^{*} \\
& P_{k}^{-}=\sum_{i=0}^{2 L} W_{i}^{(c)}\left(\mathcal{X}_{i, k \mid k-1}^{*}-\hat{x}_{k}^{-}\right)\left(\mathcal{X}_{i, k \mid k-1}^{*}-\hat{x}_{k}^{-}\right)^{T}+R^{v} \\
& \mathcal{X}_{k \mid k-1}=\left[\begin{array}{lll}
\mathcal{X}_{k \mid k-1}^{*} & \mathcal{X}_{0, k \mid k-1}^{*}+\gamma \sqrt{R^{v}} & \mathcal{X}_{0, k \mid k-1}^{*}-\gamma \sqrt{R^{v}}
\end{array}\right] \\
& \mathcal{Y}_{k-1}=H\left(\mathcal{X}_{k-1}\right) \\
& \hat{y}_{k}^{-}=\sum_{i=0}^{2 L} W_{i}^{(m)} \mathcal{Y}_{i, k \mid k-1}^{*}
\end{aligned}
$$

Measurement-update:

$$
\begin{aligned}
P_{\tilde{y_{k}} \tilde{y_{k}}} & =\sum_{i=0}^{2 L} W_{i}^{(c)}\left(\mathcal{Y}_{i, k \mid k-1}-\hat{y}_{k}^{-}\right)\left(\mathcal{Y}_{i, k \mid k-1}-\hat{y}_{k}^{-}\right)^{T}+R^{n} \\
P_{x_{k} y_{k}} & =\sum_{i=0}^{2 L} W_{i}^{(c)}\left(\mathcal{X}_{i, k \mid k-1}-\hat{x}_{k}^{-}\right)\left(\mathcal{Y}_{i, k \mid k-1}-\hat{y}_{k}^{-}\right)^{T} \\
\mathcal{K}_{k} & =P_{x_{k} y_{k}} P_{\tilde{y_{k}} \tilde{y}_{k}}^{-1} \\
\hat{x}_{k} & =\hat{x}_{k}^{-}+\mathcal{K}_{k}\left(y_{k}-\hat{y}_{k}^{-}\right) \\
P_{k} & =P_{k}^{-}+\mathcal{K}_{k} P_{\tilde{y_{k}} \tilde{y_{k}}} \mathcal{K}_{k}^{T}
\end{aligned}
$$

\subsection{Simulation results for parameter estimation}

Tab. 4 lists the identified state variables and parameters. Parameter identification is done for RhSt, DhwSt, PV/T, and for the building model parameters of the SFH100. It is performed during the normal building operation.

Tab. 3: Simulations settings

\begin{tabular}{ll} 
Parameter & Value \\
\hline Measurement interval for identification & $0.5 \mathrm{~h}$ \\
Spread of sigma points $\alpha$ & $1 \mathrm{e}-3$ \\
Forgetting factor $\Theta$ & $1 \mathrm{e}-4 \ldots 1 \mathrm{e}-3$ \\
Optimization interval (MPC) & $1 \mathrm{~h}$ \\
Prediction horizon (MPC) & $8 \mathrm{~h}$ \\
Identification duration & $2 \ldots . .50$ days \\
\hline
\end{tabular}


P. Bolt et. al. / EuroSun 2018 / ISES Conference Proceedings (2018)

Tab. 4: Measurement $y$, identified parameters, disturbance $d$ and inputs u of the model.

\begin{tabular}{|c|c|c|}
\hline Variable & Description & Range/Unit \\
\hline $\mathrm{y}_{1}, \mathrm{y}_{2}, \mathrm{y}_{3}, \mathrm{y}_{4}$ & temperatures of the RhSt (4 layers) & ${ }^{\circ} \mathrm{C}$ \\
\hline $\mathrm{y}_{5}, \mathrm{y}_{6}, \mathrm{y}_{7}, \mathrm{y}_{8}$ & temperatures of the DwSt (4 layers) & ${ }^{\circ} \mathrm{C}$ \\
\hline $\mathrm{y}_{9}$ & temperature of the PVT & ${ }^{\circ} \mathrm{C}$ \\
\hline $\mathrm{y}_{\text {con }}$ & temperature of the concrete & ${ }^{\circ} \mathrm{C}$ \\
\hline yroom & temperature of the room & ${ }^{\circ} \mathrm{C}$ \\
\hline $\mathrm{Cm}_{1 \mathrm{SFH} 100}$ & thermal mass & $\mathrm{J} / \mathrm{K}$ \\
\hline $\mathrm{Cm}_{2} \mathrm{SFH} 100$ & thermal mass & $\mathrm{J} / \mathrm{K}$ \\
\hline $1 / \mathrm{R}_{1 \mathrm{SFH} 100}$ & thermal conduction to the ambient & $\mathrm{W} / \mathrm{K}$ \\
\hline $1 / \mathrm{R}_{2} \mathrm{SFH} 100$ & thermal conduction between thermal masses & $\mathrm{W} / \mathrm{K}$ \\
\hline $1 / \mathrm{R}_{3} \mathrm{SFH} 100$ & thermal conduction to soil & $\mathrm{W} / \mathrm{K}$ \\
\hline $\mathrm{K}_{1 \mathrm{SFH} 100}$ & solar factor & $\mathrm{m}^{2}$ \\
\hline $\mathrm{Cm}_{\mathrm{ST} 1}$ & thermal mass of the storage & $\mathrm{J} / \mathrm{K}$ \\
\hline $\mathrm{Cm}_{\mathrm{ST2} 2}$ & thermal mass of the storage & $\mathrm{J} / \mathrm{K}$ \\
\hline $1 / \mathrm{Cm}_{\mathrm{PVT}}$ & thermal mass (inverse) & $\mathrm{K} / \mathrm{J}$ \\
\hline $\mathrm{P}_{1}$ & lumped parameter & $\left(\mathrm{K} \mathrm{m}^{2}\right) / \mathrm{J}$ \\
\hline $\mathrm{P}_{2}$ & lumped parameter & $1 / \mathrm{s}$ \\
\hline $\mathrm{d}_{\text {dist }}$ & internal gains (unkown) & $\mathrm{W} / \mathrm{m}^{2}$ \\
\hline $\mathrm{d}_{\mathrm{TAmb}}$ & ambient temperature & ${ }^{\circ} \mathrm{C}$ \\
\hline$d_{\text {solar }}$ & solar radiation & $\mathrm{W} / \mathrm{m}^{2}$ \\
\hline $\mathrm{u}_{\text {pump HP1 }}, \mathrm{u}_{\text {pump HP2 }}$ & control input pumps & $\{0,1\}$ \\
\hline $\mathrm{u}_{\mathrm{T} \text { sup } \mathrm{ST} 1,} \mathrm{u}_{\mathrm{T} \text { ret } \mathrm{ST} 1,} \mathrm{u}_{\mathrm{T} \text { sup ST2},} \mathrm{u}_{\mathrm{T}}$ ret ST2 & supply and return temperature heat $\mathrm{ST}$ & ${ }^{\circ} \mathrm{C}$ \\
\hline $\mathrm{u}_{\mathrm{T} \text { sup } \mathrm{PVT}}, \mathrm{u}_{\mathrm{T} \text { ret } \mathrm{PVT}}$ & supply and return temperature PVT & ${ }^{\circ} \mathrm{C}$ \\
\hline $\mathrm{u}_{\mathrm{T} \text { sup SFH100, }}, \mathrm{u}_{\mathrm{T} \text { ret } \mathrm{SFH} 100}$ & supply and return temperature SFH & ${ }^{\circ} \mathrm{C}$ \\
\hline
\end{tabular}

For the identification of the building model, we presuppose very little knowledge of the actual parameter values. Twelve simulation runs are executed with randomly chosen initial values. Identification of the building model parameters (SFH 100) for randomly chosen initial conditions is shown in Fig. 4. Within ca. 20 days all parameter estimates - with one exception - converge. The estimated parameters are lumped parameters, thus only indirect verification of the estimation is possible. Fig. 3 shows the error for room and concrete temperatures for one of the runs.

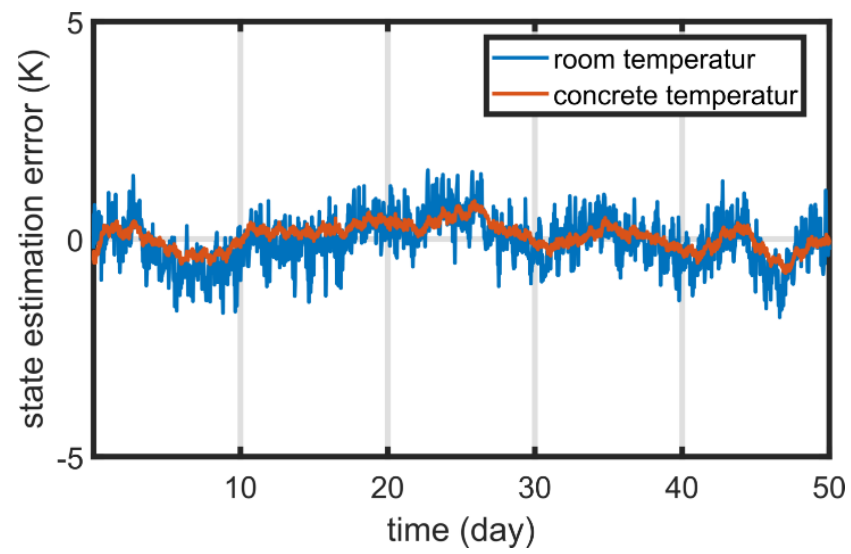

Fig. 3: Error between estimation and measured room and concrete temperature using UKF. The temperatures depend on the estimated parameters and can be measured directly. 

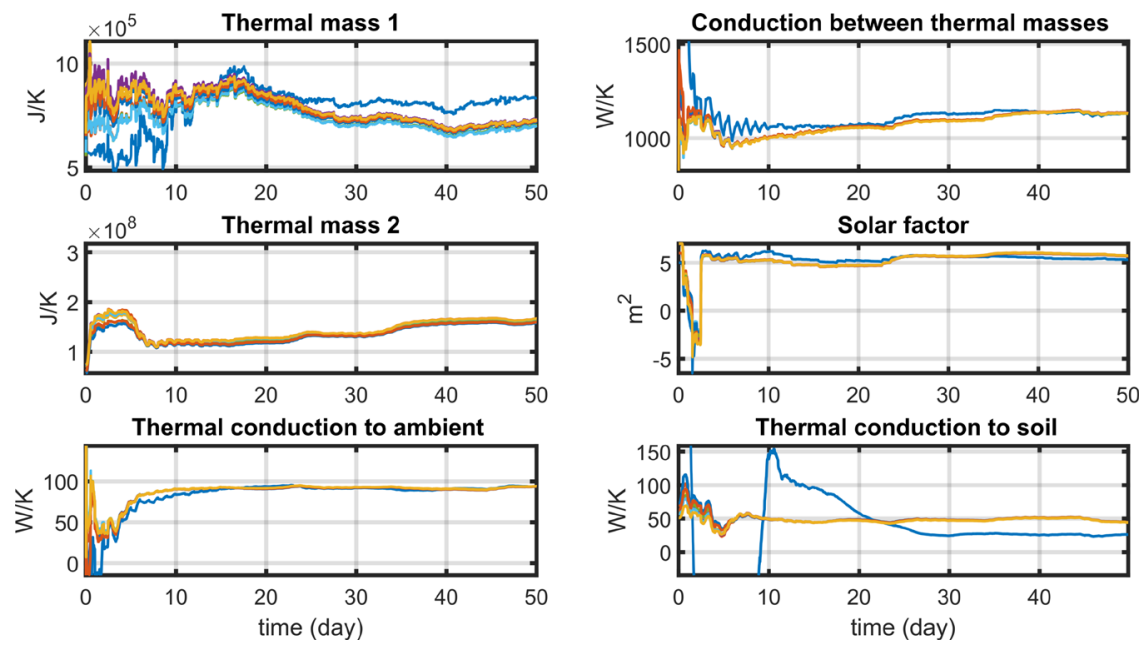

Fig. 4: Identification of the building model parameters (SFH 100) with different initial conditions during ordinary operation of the heating system. Several simulation runs with different initial guesses are shown. Within ca. 20 days all parameters converge, except in one of the runs (blue line) which failed to converge. The parameters are lumped parameters, thus they cannot be compared to "exact" values.

The identification of the heating system parameters (RhSt, DhwSt, PV/T) is shown in Fig. 5 and Fig. 6. Convergence is faster, than for the parameters in Fig. 4, because a good initial estimate is usually available.
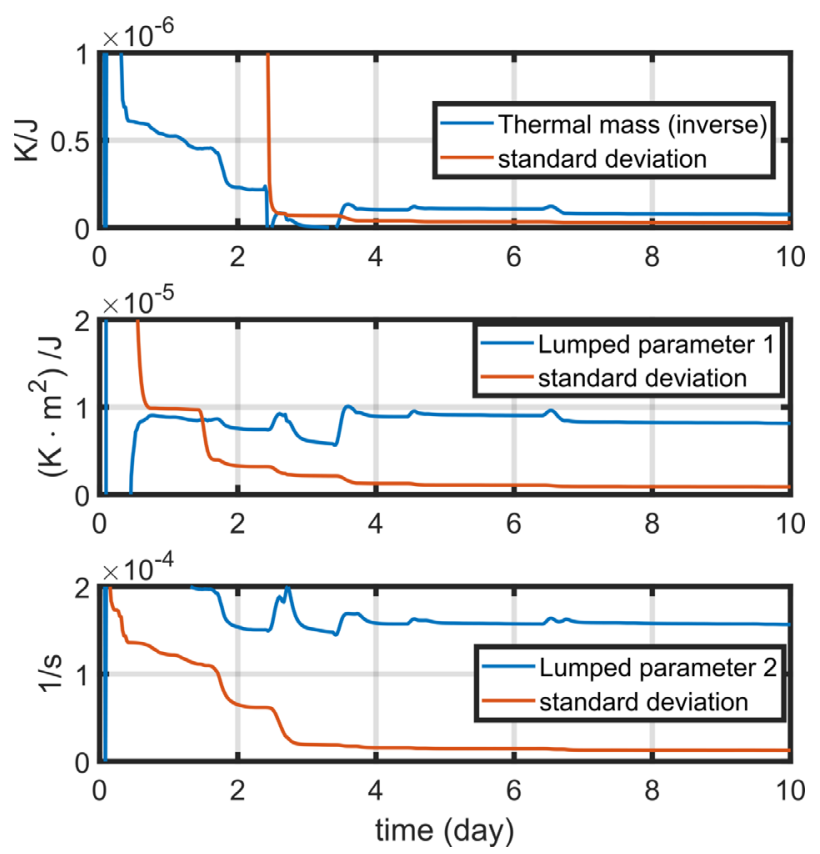

Fig. 5: Identification for the parameters. Compared to the identification of DhwSt and RhSt it takes a bit longer to reach a stable set of parameters. This is because the identification only can run when some excitation (irradiation sun) is active.
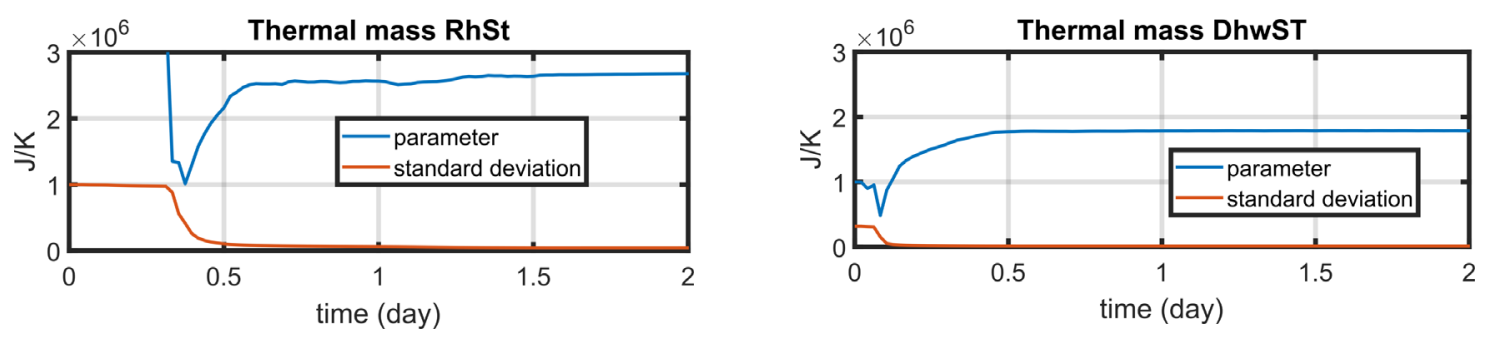

Fig. 6: Identification for the thermal mass of DhwSt and RhSt. Within ca. 0.5 days, the parameters converge with a relative error of 0.14 and 0.22 respectively. 


\section{Results for overall performance}

All presented results refer to annual simulation with synthetic weather data (averaged over several years) for Zurich, Switzerland, as provided by METEONORM 7 (Meteotest, 2013). We compare MPC to a Rule Based Controller (RBC) in terms of performance coefficient, self-consumption and self-sufficiency.

\subsection{Performance coefficient, self-consumption and self-sufficiency}

The performance coefficient is by definition

$$
J:=\int \frac{\dot{Q}_{t h}}{\dot{E}_{\text {ext }}} d t
$$

where $\dot{Q}_{t h}$ is the total useable thermal energy supplied for hot water supply and heating and $\dot{E}_{\text {ext }}$ is the imported electrical energy. We calculate the annual $J$ and monthly $J_{\text {monthly }}$ performance coefficient.

The system has a performance factor of $J=6.85$ for the MPC and $J=4.24$ for the RBC (Tab. 5). During the heating season the MPC achieves for the coldest months, January and December, a performance factor $J_{\text {monthly }}=4.9$ and 5 , while $\mathrm{RBC}$ reaches $J_{\text {monthly }}=3.8$ and 4.0 (Fig. 7).

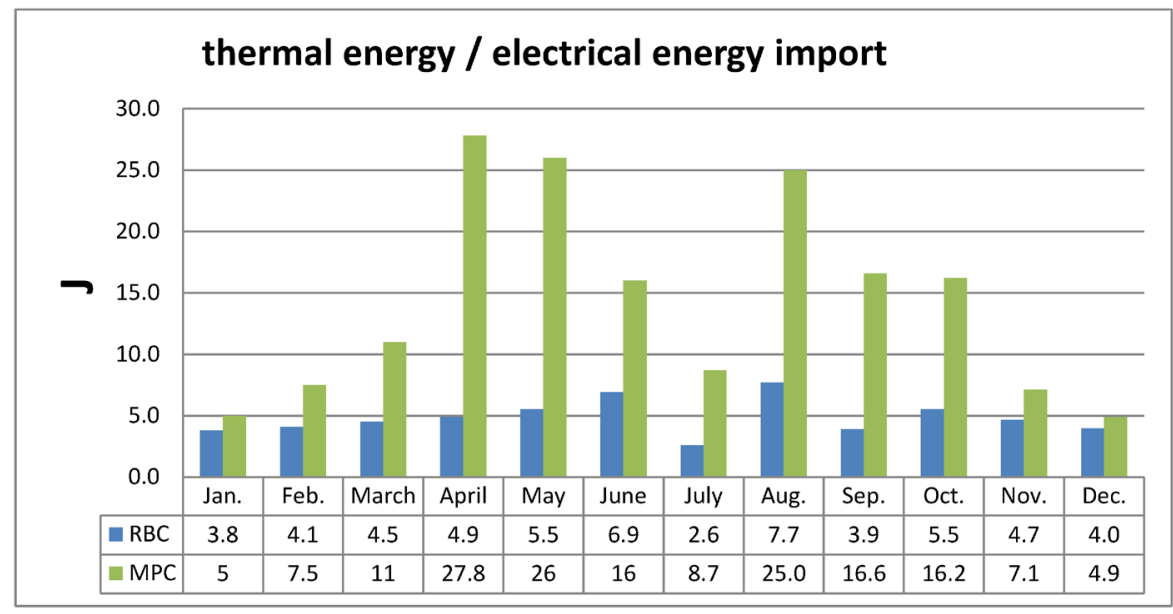

Fig. 7: Monthly performance factors $\mathrm{J}_{\text {monthly }}$ for $\mathrm{RBC}$ and MPC resulting from a realistic simulation with Zurich weather data.

Tab. 5: Result overview. RBC and MPC deliver roughly the same amount of heat and produce the same amount of energy, but MPC can use a much larger share of the produced energy on site.

\begin{tabular}{|c|c|c|c|}
\hline & Unit & $\mathrm{RBC}$ & MPC \\
\hline \multicolumn{4}{|l|}{ heating circuit } \\
\hline heating energy & $\mathrm{kWh}$ & 17119 & 17250 \\
\hline energy DHW & $\mathrm{kWh}$ & 2065 & 2065 \\
\hline thermal energy out (no cooling) & kWh & 19184 & 19316 \\
\hline cooling & $\mathrm{kWh}$ & 1371 & 1370 \\
\hline \multicolumn{4}{|l|}{ electricity } \\
\hline produced PV & $\mathrm{kWh}$ & 9508 & 9641 \\
\hline consumed WPs \& pumps & $\mathrm{kWh}$ & 5530 & 6466 \\
\hline exported & $\mathrm{kWh}$ & 8505 & 5994 \\
\hline imported & kWh & 4527 & 2819 \\
\hline Performance coefficient $\mathbf{J}$ & - & 4.24 & 6.85 \\
\hline
\end{tabular}

For self-consumption (eq. 10) and self-sufficiency (eq. 11) we use the terms as described by (Luthander et al., 2015). The meaning of the variables are self-consumption ratio $R_{\text {con }}(-)$, self-sufficiency ratio $R_{\text {suf }}(-)$, self-consumption of on-site PV electricity $E_{\text {self }}(\mathrm{kWh})$, electricity consumption $E_{\text {con }}(\mathrm{kWh})$, electricity import form the grid $E_{\text {grid }}(\mathrm{kWh})$ and on-site PV electricity produced $E_{\text {prod }}(\mathrm{kWh})$. 


$$
\begin{aligned}
& R_{\text {con }}=\frac{E_{\text {self }}}{E_{\text {prod }}}=\frac{E_{\text {con }}-E_{\text {grid }}}{E_{\text {prod }}} \\
& R_{\text {suf }}=\frac{E_{\text {self }}}{E_{\text {con }}}=\frac{E_{\text {con }}-E_{\text {grid }}}{E_{\text {con }}}=1-\frac{E_{\text {grid }}}{E_{\text {con }}}
\end{aligned}
$$

With MPC, the system consumes $R_{c o n}=56 \%$ of the generated PVT electrical energy in the course of one year and reaches a self sufficiency $R_{\text {suf }}=38 \%$ (Fig. 8, Fig. 9). With RBC, the self-consumption ratio is approximately $10 \%$ based only on coincidence and the self-sufficiency is $18 \%$. During the heating season from October to April the MPC achieves a PV self-consumption between $47 \%$ and $69 \%$. The worst month with respect to self-consumption is April and the best month is December.

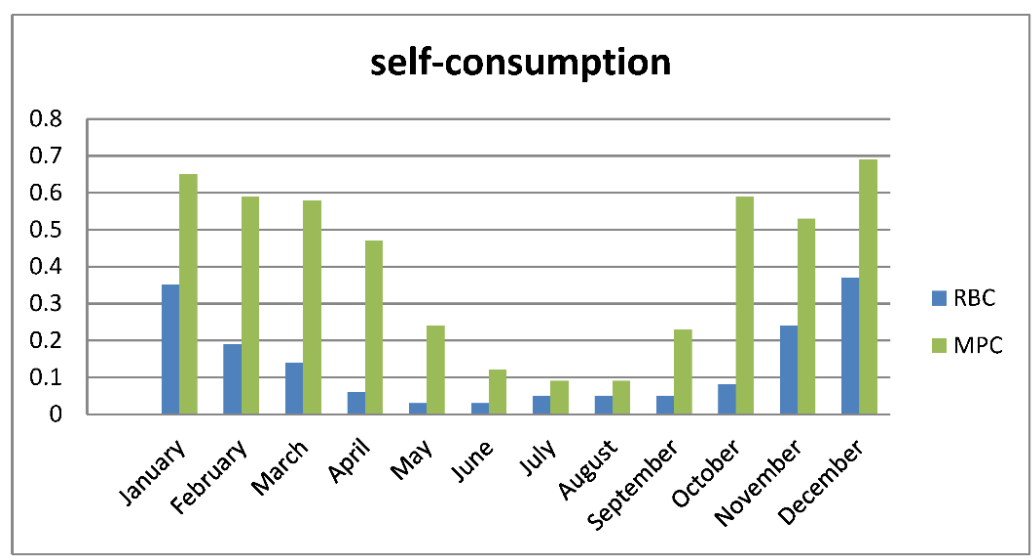

Fig. 8: Monthly self-consumption.

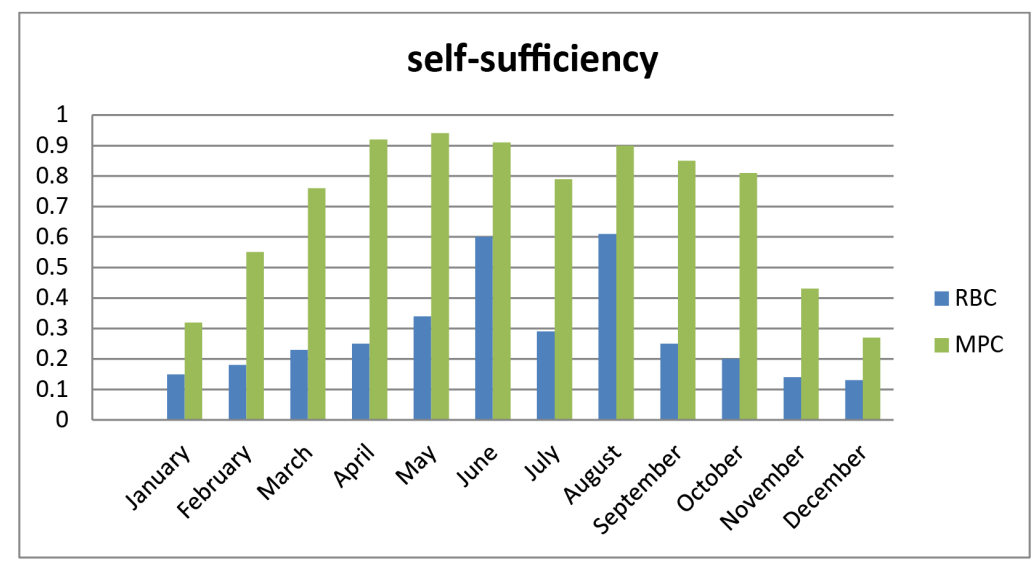

Fig. 9: Monthly self-sufficiency.

\subsection{Discussion}

There are two main reasons for optimizing the self-consumption. First, there is an economical reward for the consumer in case of low feed-in prices and high purchase prices. With variable electricity prices, the potential is even greater (Zogg et al., 2016). Second, grid-connected PV power plants may cause unwanted disturbances to the electricity grid.

Maximizing self-consumption and efficient operation of the HP are contradictory: maximizing the self-consumption implies heating up the storage while on-site PV electricity is available. The consequence are higher temperature lifts and thus a decreased efficiency of the HP. The HP still operates with maximum efficiency while there is no PV/T electricity generation.

Other common seasonal performance figures, such as the Seasonal Performance Factor $(S P F+)$ define the overall energy efficiency of the system as the quotient of overall useful energy output and the overall driving final energy input (Malenkovic et al., 2013) without distinction between imported and locally produced electrical energy. However if self-consumption should play a role in the optimization, this distinction is reasonable. 
In our setup, we find values of $\mathrm{SPF}+=3.2$ for $\mathrm{MPC}$ and $\mathrm{SPF}+=3.7$ for $\mathrm{RBC}$.

\section{Conclusion and Outlook}

In an annual simulation run with Zurich weather data, and two consumers (one SFH45 and one SFH100), a performance factor of $\mathrm{J}=6.85$ was reached. Economically this is a very competitive value (i.e. running costs are competitive). Similar systems like (Daniel Philippen, 2015) reach a ratio up to 7 for SFH45 and up to 3.5 for SFH100. Commissioning and maintenance costs are kept low by the continuous parameter identification, which ensures, that the model remains accurate over time, even in the presence of varying system parameters. Even with automatic adaptation of system parameters, the required modelling effort remains considerable. Model free, possibly heuristic or self-learning, optimal control concepts are therefore a very interesting future research objective.

\section{Acknowledgements}

The present work has been funded by the Swiss Commission for Technology and Innovation (KTI). The project is a collaboration between RINO Electronics AG, Jansen AG and the Zurich University of Applied Sciences (ZHAW).

\section{References}

Ashouri, A., Stadler, P., and Maréchal, F. (2015). Day-ahead promised load as alternative to real-time pricing. In 2015 IEEE International Conference on Smart Grid Communications (SmartGridComm), pp. 551-556.

Bianchi, M. (2006). Adaptive Modellbasierte Prädiktive Regelung einer Kleinwärmepumpenanlage. ETH Zürich.

Carigiet, F., Baumgartner, F., Sutterlueti, J., Allet, N., Pezzotti, M., and Haller, J. (2014). Energy rating based on thermal modelling of five different PV technologies. In Proceedings of the 29th European Photovoltaic Solar Energy Conference, Amsterdam, The Netherlands, pp. 22-26.

Daniel Philippen, D.C. (2015). High-Ice System development for high solar thermal gains with ice storage and heat pump.

Dubey, S., Sarvaiya, J.N., and Seshadri, B. (2013). Temperature Dependent Photovoltaic (PV) Efficiency and Its Effect on PV Production in the World - A Review. Energy Procedia 33, 311-321.

Fritzson, P., and Engelson, V. (1998). Modelica - A unified object-oriented language for system modeling and simulation. In ECOOP'98 - Object-Oriented Programming, (Springer, Berlin, Heidelberg), pp. 67-90.

Fux, S.F. (2013). Optimal energy management and component sizing of a stand-alone building energy system. ETH Zurich.

Gurobi Optimization, LLC (2018). Gurobi Optimizer Reference Manual.

Haller, M.Y., Dott, R., Ruschenburg, J., Ochs, F., and Bony, J. (2013). The Reference Framework for System Simulations of the IEA SHC Task 44/HPP Annex 38 Part A: General Simulation Boundary Conditions (Citeseer).

Haykin, S.S. (2001). Kalman Filtering and Neural Networks (New York, NY, USA: John Wiley \& Sons, Inc.).

Julier, S.J., Uhlmann, J.K., and Durrant-Whyte, H.F. (1995). A new approach for filtering nonlinear systems. In American Control Conference, Proceedings of the 1995, pp. 1628-1632 vol.3.

Lofberg, J. (2004). YALMIP: A toolbox for modeling and optimization in MATLAB. In Computer Aided Control Systems Design, 2004 IEEE International Symposium On, (IEEE), pp. 284-289.

Luthander, R., Widén, J., Nilsson, D., and Palm, J. (2015). Photovoltaic self-consumption in buildings: A review. Applied Energy 142, 80-94.

Maasoumy, M., Razmara, M., Shahbakhti, M., and Vincentelli, A.S. (2014). Handling model uncertainty in model predictive control for energy efficient buildings. Energy and Buildings 77, 377-392. 
P. Bolt et. al. / EuroSun 2018 / ISES Conference Proceedings (2018)

Malenkovic, I., Eicher, S., and Bony, J. (2013). Definition of main system boundaries and performance figures for reporting on SHP systems. Report Deliverable B 1 .

Meteotest (2013). Global Meteorological Database for Engineers, Planners and Education (Bern, Switzerland).

Peter S. Maybeck (1979). Stochastic Models Estimation And Control VOL 1.

Peter S. Maybeck (1982). Stochastic Models Estimation And Control VOL. 2.

Radecki, P., and Hencey, B. (2015). Online Model Estimation for Predictive Thermal Control of Buildings. ArXiv:1601.02947 [Cs].

Ralf Dott, M.Y.H. (2014). The Reference Framework for System Simulations of the IEA SHC Task 44 / HPP Annex 38 Part B: Buildings and Space Heat Load.

Schneider, R., and Georgakis, C. (2013). How To NOT Make the Extended Kalman Filter Fail. Industrial \& Engineering Chemistry Research 52, 3354-3362.

Serale, G., Fiorentini, M., Capozzoli, A., Bernardini, D., and Bemporad, A. (2018). Model Predictive Control (MPC) for Enhancing Building and HVAC System Energy Efficiency: Problem Formulation, Applications and Opportunities. Energies 11, 631.

Sturzenegger, D. (2014). Model predictive building climate control: Steps towards practice. Doctoral Thesis. ETH Zurich.

Wan, E.A., and Merwe, R.V.D. (2000). The unscented Kalman filter for nonlinear estimation. In Proceedings of the IEEE 2000 Adaptive Systems for Signal Processing, Communications, and Control Symposium (Cat. No.00EX373), pp. 153-158.

Wetter, M., and van Treeck, C. (2017). IEA EBC Annex 60: New Generation Computing Tools for Building and Community Energy Systems. The Regents of the University of California and RWTH Aachen University.

Wimmer, R. (2004). Regelung einer Wärmepumpenanlage mit Model Predictive Control. ETHZ. 